\title{
Multi-parameter Grain Analysis Model based on BP Neural Network
}

\author{
Yufeng Zhuang1, a , Runfa Lu 2 b \\ ${ }^{1}$ School of Automation, Beijing University of Posts and Telecommunications, Beijing 100876 , \\ China; \\ ${ }^{2}$ School of Automation, Beijing University of Posts and Telecommunications, Beijing 100876, \\ China.
}

azhuangyf@bupt.edu.cn, blurunfa@163.com

\begin{abstract}
During the process of grain storage, changes in seasons and weather will cause changes in the temperature and humidity of the granary, which will affect the occurrence of pests in the grain pile and the possibility of condensation, leading to problems such as grain deterioration and water loss. In this paper, based on the Bp neural network model, parameters such as temperature, humidity, moisture, warehouse temperature and warehouse moisture are used as input factors of the model to construct a multi-parameter grain analysis model. Through comparative analysis, the model optimization parameters are selected, and the trained neural network model is convenient for food administrators to obtain food storage status more intuitively.
\end{abstract}

Keywords: Neural Networks; Grain situation analysis; Multi-parameter grain situation.

\section{Introduction}

According to the "Twelfth Five-Year Plan", the grain industry has been steadily improved in its grain testing and emergency support capabilities. In the latest "13th Five-Year Plan", one of the important development goals proposed by the state: significantly improving food situation warning and emergency support capabilities [1]. Through the construction of intelligent grain storage, hundreds of sensors and high-definition cameras are arranged in the granary to remotely monitor changes in parameters such as temperature, humidity, moisture, and pests in the cabin. Once the temperature or other parameters are too high, an alarm will be issued and the pattern of manual marking will be replaced [2]. However, this simple discriminant model is often affected by factors such as temperature and humidity of the weather, leading to misjudgment of the model and unable to completely get rid of the manual on-site confirmation. In summary, the grain storage environment is a complex system consisting of various factors such as temperature, humidity, moisture and weather. BP neural network has strong adaptability and self-learning ability to complex nonlinear systems, and can approximate nonlinear functions with arbitrary precision. Therefore, this paper uses BP neural network to construct a grain analysis model that can be applied to multiple parameters.

\section{Grain Situation Analysis Model based on BP Neural Network}

\subsection{BP Neural Network}

The BP neural network is a multi-layer feedforward network trained by the error back propagation algorithm. Its main feature is the forward transmission of signals, and the error is reversely propagated. Figure 1 shows the BP neural network diagram of three input nodes, where input layer is input information and output layer is output information. In BP neural network, there is no feedforward loop between each layer, and each node in the same layer There is no connection between them. The input information passes through the hidden layer and is output through the output layer. If the error between the output result and the expected result is greater than the set value, the weight of each node on the transmission route is adjusted according to the error. The most important advantage of neural networks over other nonlinear methods is that the neural network can be globally approximated and highly accurate. At the same time, the modeling of neural networks does not require prior assumptions, which are largely determined by the characteristics of the data. BP neural networks can describe 
complex, nonlinear, and uncertain systems. If the desired output cannot be obtained at the output layer, it is transferred to the backpropagation, and the error signal is returned along the original connection path. By modifying the weight of each neuron, the error signal is minimized [3]

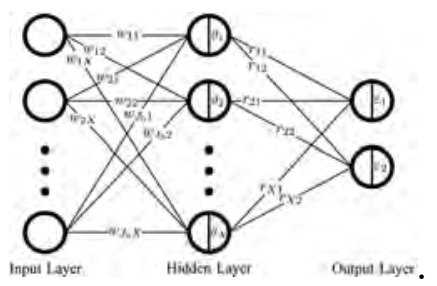

Fig. 1 BP neural network structure

\subsection{BP Neural Network Algorithm Description}

The BP algorithm is a supervised learning algorithm belonging to the gradient descent algorithm. Assuming that the input of the neuron $\mathrm{P}=\left\{p_{1}, p_{2}, \ldots p_{n}\right\}$,implicit layer neuron $\mathrm{S}=\left\{s_{1}, s_{2} \ldots, s_{k}\right\}$ and output layer neuron $\mathrm{A}=\left\{a_{1}, a_{2}, \ldots a_{m}\right\} . W_{n, k}^{1}$ represents the connection weight of the $\mathrm{nth}$ neuron of the input layer to the kth neuron of the hidden layer and $W_{k, m}^{2}$ represents the connection weight of the kth neuron of the hidden layer to the mth neuron of the output layer. $b_{s_{k}}^{1}$ represents the threshold of the $S_{k}$ neuron in the hidden layer and $b_{a_{m}}^{2}$ represents the threshold of the $a_{m}$ phase of the output layer. $f^{1}$ is the activation function of the hidden layer. $f^{2}$ is the activation function of the output layer. Among them $W_{n, k}^{1}, W_{k, m}^{2}, b_{s_{k}}^{1}, b_{a_{m}}^{2} \in(-1,1)$.

(1) Forward propagation

$$
\begin{array}{r}
s_{k}=f^{1}\left(p_{1} * W_{1, k}^{1}+p_{2} * W_{2, k}^{1}+\ldots+p_{n} * W_{n, k}^{1}+b_{s_{k}}^{1}\right) \\
a_{m}=f^{2}\left(s_{1} * W_{1, m}^{2}+s_{2} * W_{2, m}^{2}+\ldots+s_{k} * W_{k, m}^{2}+b_{a_{m}}^{2}\right)
\end{array}
$$

(2) Cost function

Through forward transfer, we can get the output of each neuron in the output layer $a_{m}$. Define the square sum error function as follows

$$
E_{p}=\sum_{m=1}^{m} \frac{1}{2}\left(a_{m}-t_{m}\right)^{2}
$$

Total error of the network under the input of all samples $\mathrm{N}$

$$
E_{N}=\sum_{p=1}^{N} E_{p}
$$

(3) Back propagation

The error propagates back to the layer from the output layer. The weight of each layer is updated by the gradient descent method

$$
w \rightarrow w^{\prime}=w-\eta \nabla E_{p}(w)
$$

$\eta$ is the step size of each update, also called the learning factor, and $\nabla E_{p}(\mathrm{w})$ is the partial derivative of the output deviation of the $\mathrm{p}$-th sample input to a certain layer of weight. The same formula for updating the threshold

$$
b \rightarrow b^{\prime}=b-\eta \nabla E_{p}(b)
$$


Through the above-mentioned method, the correction is recalculated until the error change rate satisfies the preset value or the number of cycles is greater than the threshold value, and the trained BP neural network is obtained.

\subsection{Improvement of Gradient Descent based on Momentum Method}

Momentum method changes the formula for updating weights and thresholds during backpropagation

$$
\begin{gathered}
w \rightarrow w^{\prime}=\mu w-\eta \nabla E_{p}(w) \\
b \rightarrow b^{\prime}=\mu b-\eta \nabla E_{p}(b)
\end{gathered}
$$

$\mu$ is the momentum factor, $\mu \in(0,1)$. In the process of backpropagation, if the set learning factor $\eta$ is too small, the smaller the change amount of the weight from the last iteration to the next generation, the smoother the trajectory space, the easier it is to approach the minimum value, which is to reduce Learning rate at the cost. On the other hand, if the learning factor $\eta$ is too large to speed up the learning rate, it is easy to make the weight change unstable (oscillation), and the increase of the momentum factor can not only ensure the learning rate, but also avoid the occurrence of oscillation [4].

\section{BP Neural Network Network Parameters}

\subsection{Input Factor}

$\mathrm{n}$ the previous literature research, it was found that the literature of the same BP neural network in the past mostly used the temperature, humidity, or only temperature of the grain pile as an input factor. In this paper, in addition to grain temperature and humidity, it also increases the moisture of the grain, the temperature in the warehouse, the humidity, the temperature of the atmosphere, and the humidity as an input factor. Due to the vastness of China's vast territory, according to the literature of the National Academy of Food Science, China is divided into seven regions. In this paper, the model training data source and the single grain of the same grain depot, thus eliminating the influence of the region on the model.

The influence of temperature and humidity factor is mainly reflected in the probability of occurrence and development of pests. The proper temperature and humidity environment is conducive to the occurrence and development of certain pests, which leads to food pests. The water factor is one of the important factors affecting the quality of food. The moisture and humidity are too large, which is likely to cause food condensation and mildew. In the literature [5] on the "Measurement of Balanced Moisture in Wheat and Intelligent Cooling and Ventilation Test in Real Warehouse" [5], it was found that water is calculated by the load-bearing method, and then the grain of different varieties is based on temperature, humidity, atmospheric temperature, humidity, etc. The parameters are fitted to the formula to obtain the fitting formula of the water as the prediction of the future moisture.

Therefore, in this paper, the four influence factors of atmospheric temperature, humidity, and temperature and humidity in the warehouse are added, which can well reflect the influence of these factors on the food situation, and after training the model, the same data is only A neural network trained with temperature and humidity factors is used as a comparison.

\subsection{Output Factor}

This paper sets the grain situation into three main states: normal grain conditions, abnormal grain conditions, and dangerous food conditions. Therefore the output layer uses a single neuron.

\subsection{Number of Hidden Layers}

For the BP neural network, there is no theoretical formula for determining the number of hidden layers. It is necessary to test the empirical formula using multiple trial and error methods until the 
number of suitable hidden layer neurons is determined [6]. The following formula is the empirical formula used:

$$
k=\sqrt{n+m}+a
$$

Where $\mathrm{k}$ is the number of hidden layers, $\mathrm{n}, \mathrm{m}$ are the number of neurons in the input and output layers, respectively, and a is a constant, generally taking a constant from 1 to 10 .

\subsection{Activation Function}

The activation function is used to change the linear output to a non-linear output, allowing the entire network to approximate any nonlinear function. This paper intends to use the sigmoid function(10) and the ReLu function(11).

$$
\begin{aligned}
& f(x)=\frac{1}{1+e^{-x}} \\
& f(x)=\max (0, x)
\end{aligned}
$$

\subsection{Sample Data}

The sample data in this experiment was derived from the grain quality inspection report of Xining Grain Reserve in Qinghai Province in 2009. The results of the analysis were derived from local experts and combined with the results of the expert food system. Since all the temperature and humidity values of each sensor layout point need to be input in the original data, the average temperature is used instead in this experiment.

\subsection{Training Objectives}

According to the application case analysis related to neural networks on the market, the error rate of more than $90 \%$ can meet the requirements of use [7]. Since the data sample size and data distribution affect the generalization and prediction ability of the model, the prediction error rate set in this experiment is $85 \%$. And the sample data was disrupted and $80 \%$ of the data was randomly selected for use as training data samples and $20 \%$ was used as test samples.

\section{BP Neural Network Network Parameters}

\subsection{Number of Hidden Layer Nodes}

Two different activation functions were selected to perform two different trial and error experiments. The results are shown in the figure. When the linear rectification type activation function is selected, the optimal number of hidden layers is 15 . The optimal number of hidden layers when selecting a logical activation function is 16 . The neural network model was constructed and tested using the best number of hidden layers, respectively.

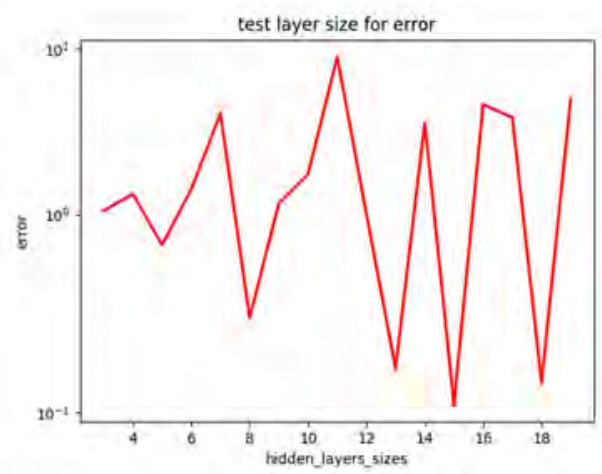

Fig.2 Implicit layer node number and loss rate 


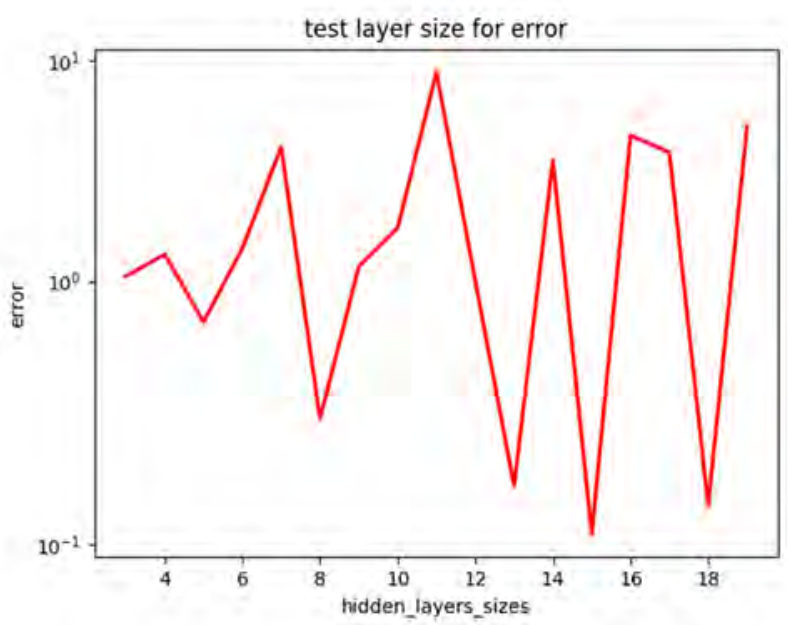

Fig.3 Implicit layer node number and loss rate relationship of sigmoid function

\subsection{Analysis of Experimental Results}

Through training, the error rate data for each training is obtained. The change threshold for setting the error rate reduction is 0.00001 . That is, when the error rate change of the next training is less than 0.00001 , it stops.

The graph of the number of training times and the error is shown in the figure, and both curves show a rapid decline. The model using the sigmod activation function has a slow convergence rate in the early stage, and it needs to train 2978 times to reach the change threshold. Compared with the model using the relu activation function, it has stabilized after training for 100 times. 955 times. Therefore, using the relu activation function as a neural network model results in a better fitting effect.

Table. 1 training result

\begin{tabular}{|cccc|}
\hline Activation function & Target error rate & Number of training & Error rate \\
\hline Sigmod & $1 \mathrm{E}-5$ & 955 & 0.08 \\
relu & $1 \mathrm{E}-5$ & 2978 & 0.22 \\
\hline
\end{tabular}

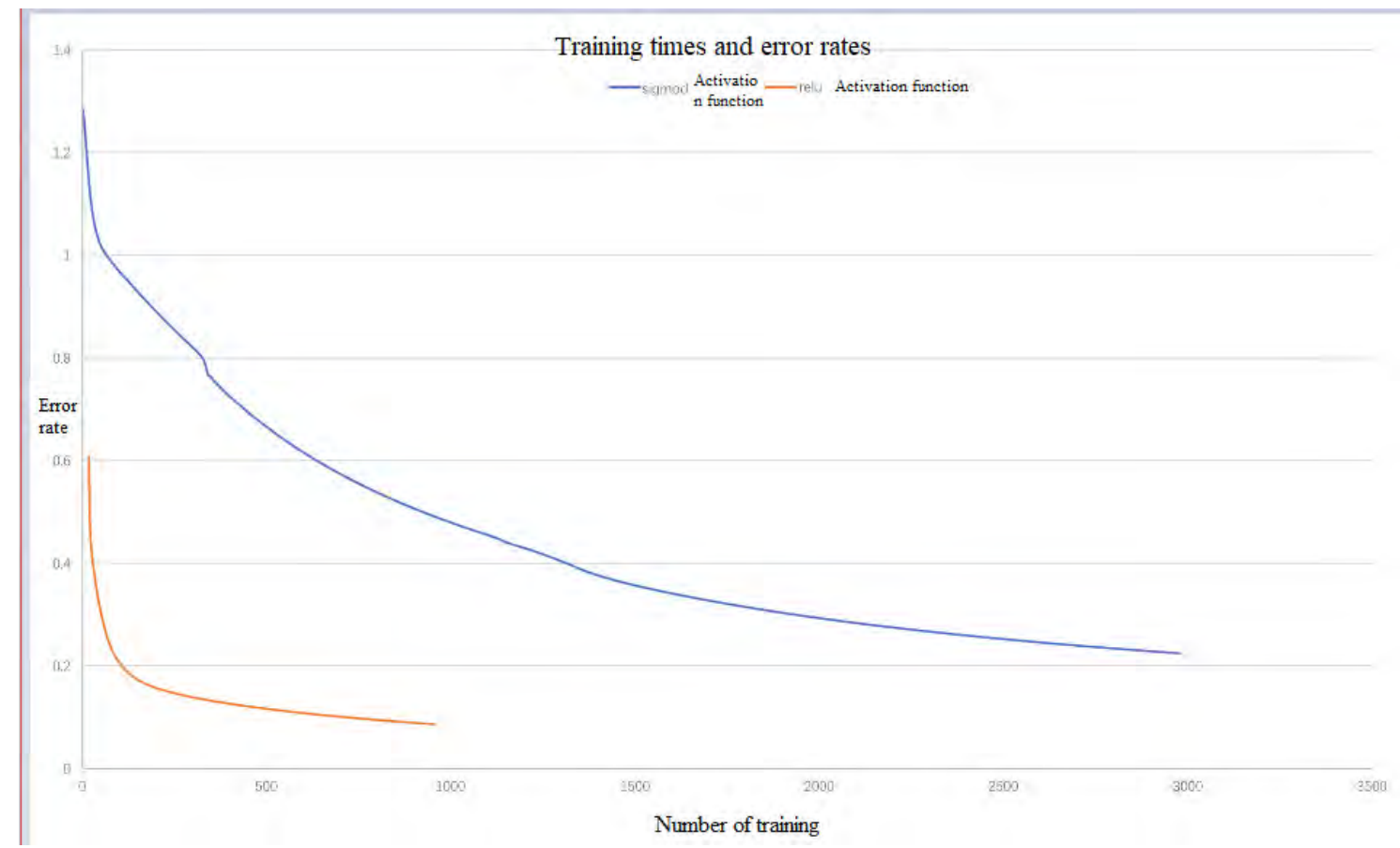

Fig.4 Training curve and error rate curve 


\subsection{Influence of Moisture, Warehouse Temperature and Humidity, and Weather on the Model}

In order to confirm the influence of moisture, temperature and humidity and weather data on the grain situation, in this experiment, a BP neural network model with input parameters only grain temperature and humidity was established. The optimal model was constructed as described above and the results are shown in the following table.

Table. 2 training result

\begin{tabular}{|cccccc|}
\hline model & $\begin{array}{c}\text { Activation } \\
\text { function }\end{array}$ & $\begin{array}{c}\text { Target error } \\
\text { rate }\end{array}$ & $\begin{array}{c}\text { Number of } \\
\text { training }\end{array}$ & $\begin{array}{c}\text { Error } \\
\text { rate }\end{array}$ & $\begin{array}{c}\text { Prediction } \\
\text { accuracy }\end{array}$ \\
\hline $\begin{array}{c}\text { Grain temperature and } \\
\text { humidity }\end{array}$ & relu & 5488 & $1 \mathrm{E}-5$ & 0.21 & 0.85 \\
Multi-parameter & relu & 955 & $1 \mathrm{E}-5$ & 0.08 & 0.98 \\
\hline
\end{tabular}

\section{Conclusion}

This paper establishes a neural network model containing 7 input variables including grain temperature, grain humidity,grain moisture, warehouse temperature and humidity, and atmospheric temperature and humidity for grain analysis. A detailed data experiment and analysis were carried out for the construction of the model and the adjustment of each parameter, and the neural network model with the highest fitting accuracy and prediction accuracy and less training times was obtained. By using the neural network model with only the temperature and humidity input variables established by the same method, comparing the two to obtain the increased parameters can increase the fitting degree and prediction accuracy of the model. Better make a clear judgment for food. Scientific and effective protection for the storage of food.

\section{References}

[1]. Anonymous. Large country granary in the Internet era: intelligent grain storage. Cereal \& Feed Industry.Vol.7(2016), p.60-60.

[2]. Xiyubo, $\mathrm{Wu}$ Wenfu, Gu Bingjie, et al. Introduction and development of grain monitoring and control system. Agriculture and technology.Vol.6 (2016) No.1, p6-7.

[3]. Lizhen, Dengzhongliang, Yangfuxing, et al. Effects of temperature and humidity on fingerprint location. Beijing.2018.

[4]. Wangjing. The Particle Swarm Optimization Algorithm Based on Ntelligent Decision System Grain Situation Control, Master Degree, Henan University of Technology, China 2011.

[5]. Li Xingjun, Wu Zidan, Ji Zhenjiang, et al. Determination of Balanced Moisture in Wheat and Intelligent Cooling and Ventilation Test of Real Warehouse. Journal of the Chinese Cereals and Oils Association.Vol.11(2017), p.94-99.

[6]. Liu Yanju, ZHOU Guang, et al. Grain Monitoring Model based on BP Neural Network.Vol.20 (2013), No.4, p.734-737.

[7]. Fu Pengcheng, Zhao Xiaojun, Li Xiaoliang, et al. Development and Application Practice of Grain Condition Analysis System. Food Storage. Vol.46(2017), No.4, p.1-4. 